\title{
Responsabilidad Social Matriz de indicadores sustentables que refleja el impacto de la forestación en tierras marginales del Partido de Bahía Blanca, Argentina ${ }^{1}$
}

Regina del Carmen

Durán*

Liliana Scoponi, M. Susana Fuertes, Marina Cordisco, Lorena Gandini, Luis Caro, Gustavo Boyer, Cristina Pitsch, Gabriela Pesce**

\footnotetext{
${ }^{1}$ Proyecto Grupo de Investigación PGI 24/C016, Ciencia y Tecnología, Argentina.

* Profesor Titular Ordinario, Departamento de Ciencias de la Administración, Universidad Nacional del Sur (UNS). Correo electrónico: rduran@ criba.edu.ar.
}

\section{Resumen}

Actualmente, se han empezado a considerar otras obligaciones que tienen las empresas para con su comunidad; se trata de aspectos vinculados con las dimensiones sociales y ambientales. Desde este contexto, nuestro trabajo tiene como punto de partida el concepto de responsabilidad social desde la noción de sustentabilidad. El objetivo es evaluar la forestación como instrumento para mejorar la sostenibilidad de los sistemas productivos tradicionales en tierras marginales del Partido de Bahía Blanca, mitigando los impactos del desarrollo no responsable de la producción primaria. Para ello, se han identificado técnicas de manejo generadoras de externalidades positivas en otras actividades agropecuarias complementarias, donde existen limitantes edafológicas, apoyándose en las tres dimensiones del concepto de sustentabilidad: ecológica, económica, sociopolítica y cultural.

** En orden correspondiente: Profesor Adjunto ordinario, UNS. estudio_scoponi@arnet.com.ar; Profesor Adjunto ordinario, UNS.sfuertes@uns.edu.ar; Ayudante ordinaria de docencia, UNS. marinacordisco@yahoo.com.ar; Ayudante ordinaria de docencia, UNS. lgandini@upso.edu.ar; Profesor Adjunto, Departamento de Agronomía, UNS. 1caro@uns.edu.ar; Ministerio de Asuntos Agrarios de la Provincia de Buenos Aires. gustavoboyer@yahoo.com.ar; Ingeniera Forestal, Universidad Nacional de Misiones. cpitsch2003@yahoo.com.ar 
De igual forma, se diseñó una matriz de evaluación y ponderación de impactos, que contempla indicadores cuantitativos y cualitativos, de cuya aplicación surge la forestación como una alternativa sustentable.

Palabras clave: desarrollo sustentable, semiárido, agroforestal.

\title{
Social Responsibility: Matrix of sustainable indicators which reflects fores- try activity impacts in marginal areas of the Partido of Bahía Blanca (Ar- gentina)
}

\begin{abstract}
Economy connects people all over the world. Nevertheless, is business success all about earning money? Recently, enterprises are concerned about aspects other than being profitable. Social and environmental dimensions are being seriously considered by them, since they are part of the same system. This research project takes the Social Responsibility concept as the key to achieve Sustainable Development. Its purpose is to evaluate forestry activity as an instrument to improve the sustainability in farming systems which are developed in marginal areas of the Partido of Bahía Blanca, in Argentina. According to this, techniques were specified, taking edaphic and climatic characteristics of the area into consideration. Also, a "matrix of assessment and measurement of impacts", with quantitative and qualitative indicators was designed. Its implementation demonstrates that forestry activity is an alternative that could be well integrated to agriculture and cattle breeding systems and therefore could bring positive externalities, considering sustainability in its three dimensions, ecology, economy sociopolitical and culture.
\end{abstract}

Key words: Sustainable development, Semi-arid, Agroforestry.

[...] Si no queremos que nuestros nietos murmuren refiriéndose a nosotros: pudiendo hacer tanto, se atrevieron a hacer tan poco. (Federico Mayor Zaragoza, ex director de la UNESCO)

\section{Introducción}

Desde la década de los ochenta, a partir de la globalización, se han observado una serie de circunstancias que han afectado (en mayor o menor medida) a la comunidad y, en especial, a las economías de los países. La apertura global ha generado una imperiosa necesidad de repensar las organizaciones desde una pers- 
pectiva más amplia, teniendo en cuenta los requerimientos de la sociedad que les ha dado origen, en virtud de la influencia que ejercen como agentes de cambio social. Las organizaciones son responsables de la forma en la que ejecutan sus decisiones para poder concretar los múltiples objetivos que persiguen. Así, pues, actualmente empiezan a considerarse otras obligaciones que tienen las empresas para con su comunidad. Son cuestiones que hacen a la actividad empresarial, pero que incorporan aspectos vinculados con las dimensiones sociales y ambientales con situaciones que visualizan erosiones importantes, acordes con el concepto actual de calidad de vida, por ejemplo, relaciones laborales y protección del medioambiente. "Un comportamiento socialmente responsable se logra cuando la estrategia, la estructura y los sistemas de la empresa reconocen explícitamente que las responsabilidades trascienden el plano puramente económico.”3 En particular, "[...] la situación ambiental genera una demanda social que nos enfrenta con el desafío de producir modificaciones en los valores, las actitudes, el modo de vida, aceptando finalmente la necesidad de propiciar un profundo cambio cultural centrado en la sustentabilidad, que incorpore la responsabilidad, individual y colectiva, hacia el cuidado y protección del medioambiente". ${ }^{4}$ Tal circunstancia, no pasa desapercibida para el sector agropecuario, el cual se ve afectado por condiciones de mercado, políticas gubernamentales e inclemencias climáticas de diversa índole que le son ajenas.

Este trabajo forma parte del Proyecto Grupos de Investigación de Ciencia y Tecnología de la Universidad Nacional del Sur (PGI 24/C016), que fue desarrollado en diferentes etapas durante los años 2006 y 2007 y que tomó como base el concepto de responsabilidad social desde la noción de sustentabilidad. El objetivo de este proyecto fue evaluar la forestación como instrumento para mejorar la sostenibilidad de los sistemas productivos tradicionales en tierras marginales del Partido de Bahía Blanca, mitigando los impactos del desarrollo no responsable de la producción primaria. Asimismo, se pretendieron identificar técnicas de manejo que generen externalidades positivas en otras actividades agropecuarias complementarias, donde existen limitantes edafológicas, apoyándose en las tres dimensiones del concepto de sustentabilidad: ecológica, económica y sociopolítica-cultural.

En un primer momento, se efectuó un diagnóstico estratégico en el que se analizaron las condiciones agroecológicas del Partido de Bahía Blanca en la Pampa

\footnotetext{
${ }^{3}$ Gallo, Miguel A. (1992), "Responsabilidades sociales dentro y fuera de la empresa. Estrategia y sociedad: responsabilidades sociales", Enciclopedia de Dirección y Administración, ORBIS, 67, p. 16.

${ }^{4}$ Kent, Patricia (2005), "Cambios en la teoría y tecnologías administrativas", en Miguel A. Vicente et al., Fundamentos de administración de organizaciones, Buenos Aires, p. 374.
} 
semiárida argentina, en términos de desarrollo agropecuario sustentable para determinar cuál era el estado de situación actual de las empresas agropecuarias.

Para este proyecto fue de suma relevancia el concepto de tierra marginal, que se refiere a suelos que se tornan improductivos para la explotación agrícola-ganadera que caracteriza a la pampa semiárida argentina y que, por esta peculiaridad, son aceptables para desarrollar la forestación como actividad complementaria. Considerando ello, en una segunda etapa, se identificaron las siguientes tierras marginales para actividades tradicionales en la región bajo estudio:

- Suelos poco profundos y con presencia de tosca.

- Suelos salinos.

- Suelos susceptibles a la erosión (eólica, hídrica).

Seguidamente, se han sugerido aquellas especies que pueden desarrollarse favorablemente en los tipos de suelos determinados: eucalyptus camaldulensis, tamarix gallica y prosopis spp. En esta tercera etapa, la metodología de trabajo integró herramientas interdisciplinarias para proponer cuatro alternativas o modelos agroforestales que se consideraron los más adecuados. El objetivo principal consistió en obtener un monte en pie y medir los beneficios o externalidades positivas que el mismo genera, sin necesidad de efectuar un manejo determinado para lograr madera de calidad. Así, se busca lograr un mejor rendimiento de las hectáreas totales o aprovechar tierras identificadas como marginales. Aplicando el criterio económico del costo ${ }^{5}$, se efectuó un análisis de costos diferenciales (incrementales) en los que se incurre, dada la complementariedad con actividades agropecuarias tradicionales, frente a la decisión de incorporar la forestación como instrumento para alcanzar la sustentabilidad del sistema, buscando un mejor aprovechamiento de los recursos y sinergia de resultados. En estos cálculos, sólo se incorporaron los subsidios ofrecidos por la Ley 25.080/98 en el nivel nacional ${ }^{6}$.

Para una mejor comprensión, se presenta un cuadro sintético con los cuatro modelos sugeridos y sus costos.

\footnotetext{
${ }^{5}$ Véase R. Durán et al. (2005), El gerenciamiento agropecuario en el siglo XXI. Bases para una competitividad sustentable, Buenos Aires, p. 237.

${ }^{6}$ También se encuentran en vigencia promociones provinciales (exención al impuesto inmobiliario Ley Provincial 7.647, art.137 del Código Rural) y beneficios municipales, como el no pago de la tasa vial.
} 
COSTO FIJO OPERATIVO POR HA, PARA CADA MODELO

\begin{tabular}{|c|c|c|c|c|c|c|c|c|}
\hline CORTINAS PROTECTORAS & \multicolumn{2}{|c|}{ Insumos } & \multicolumn{2}{|c|}{ Manode Obra } & \multicolumn{2}{|c|}{ Maquinarias } & \multicolumn{2}{|c|}{ TOTAL } \\
\hline Centros de Costos & Pesos & $\%$ & Pesos & $\%$ & Pesos & $\%$ & Pesos & $\%$ \\
\hline Preparación del Terreno & 21,90 & $3 \%$ & 0,00 & $0 \%$ & 25,71 & $3 \%$ & 47,61 & $3 \%$ \\
\hline Plantación & 638,27 & $97 \%$ & 116,19 & $53 \%$ & 23,86 & $3 \%$ & 778,31 & $48 \%$ \\
\hline Mantenimiento $\mathrm{h} / \mathrm{planta}$ lograda & 0,00 & $0 \%$ & 103,75 & $47 \%$ & 693,19 & $93 \%$ & 796,94 & $49 \%$ \\
\hline TOT A L & 660,17 & $41 \%$ & 219,94 & $14 \%$ & $7 \mathbf{7 4 2 , 7 5}$ & $46 \%$ & 1622,85 & $100 \%$ \\
\hline
\end{tabular}

\begin{tabular}{|c|c|c|c|c|c|c|c|c|}
\hline MONTE DE ABRIGO & \multicolumn{2}{|c|}{ Insumos } & \multicolumn{2}{|c|}{ Manode Obra } & \multicolumn{2}{|c|}{ Maquinarias } & \multicolumn{2}{|c|}{ TOTAL } \\
\hline Centros de Costos & Pesos & $\%$ & Pesos & $\%$ & Pesos & $\%$ & Pesos & $\%$ \\
\hline Preparación del Terreno & 499,60 & $5 \%$ & 0,00 & $0 \%$ & 110,51 & $10 \%$ & 160,11 & $6 \%$ \\
\hline Plantación & 1048,91 & $95 \%$ & 205,51 & $55 \%$ & 118,95 & $11 \%$ & 1373,37 & $53 \%$ \\
\hline Mantenimiento $\mathrm{h} / \mathrm{planta}$ lograda & 0,00 & $0 \%$ & 165,05 & $45 \%$ & 884,52 & $79 \%$ & 1049,57 & $41 \%$ \\
\hline TOTAL & 1098,51 & $43 \%$ & 370,57 & $14 \%$ & 1113,98 & $43 \%$ & 2583,06 & $100 \%$ \\
\hline
\end{tabular}

\begin{tabular}{|c|c|c|c|c|c|c|c|c|}
\hline MONTE ENERGETICO & \multicolumn{2}{|c|}{ Insumos } & \multicolumn{2}{|c|}{ Manode Obra } & \multicolumn{2}{|c|}{ Maquinarias } & \multicolumn{2}{|c|}{ TOTAL } \\
\hline Centros de Costos & Pesos & $\%$ & Pesos & $\%$ & Pesos & $\%$ & Pesos & $\%$ \\
\hline Preparación del Terreno & 69,60 & $4 \%$ & 0,00 & $0 \%$ & 756,43 & $22 \%$ & 826,03 & $13 \%$ \\
\hline Plantación & 1715,98 & $96 \%$ & 326,00 & $33 \%$ & 193,66 & $6 \%$ & 2235,64 & $36 \%$ \\
\hline Mantenimiento $\mathrm{h} / \mathrm{planta}$ lograda & 0,00 & $0 \%$ & 654,71 & $67 \%$ & 2489,61 & $72 \%$ & 3144,32 & $51 \%$ \\
\hline TOTA L & 1785,58 & $29 \%$ & 980,71 & $16 \%$ & 34339,70 & $55 \%$ & 6205,99 & $100 \%$ \\
\hline
\end{tabular}

\begin{tabular}{|c|c|c|c|c|c|c|c|c|}
\hline MONTE DE ABRIGO Y ENERGETICO & \multicolumn{2}{|c|}{ Insumos } & \multicolumn{2}{|c|}{ Manode Obra } & \multicolumn{2}{|c|}{ Maquinarias } & \multicolumn{2}{|c|}{ TOTAL } \\
\hline Centros de Costos & Pesos & $\%$ & Pesos & $\%$ & Pesos & $\%$ & Pesos & $\%$ \\
\hline Preparación del Terreno & 0,00 & $0 \%$ & 0,00 & $0 \%$ & 21,37 & $9 \%$ & 21,37 & $2 \%$ \\
\hline Plantación & 480,00 & $79 \%$ & 12,28 & $20 \%$ & 45,01 & $18 \%$ & 537,29 & $59 \%$ \\
\hline Mantenimiento $\mathrm{h} / \mathrm{planta}$ lograda & 124,00 & $21 \%$ & 49,12 & $80 \%$ & 180,04 & $73 \%$ & 353,16 & $39 \%$ \\
\hline TOTA L & 604,00 & $66 \%$ & 61,40 & $7 \%$ & 246,42 & $27 \%$ & 911,82 & $100 \%$ \\
\hline
\end{tabular}

\section{Forestación y sustentabilidad}

Los nuevos enfoques de gestión de los establecimientos agropecuarios se orientan hacia una concepción más global de la empresa y su entorno. Dentro de esta vertiente, que reúne una serie de diversos aspectos íntimamente vinculados, cobra relevancia el concepto de sustentabilidad. En el espacio de la sustentabilidad lo que se busca es mantener producciones regularizadas y bajo economías rentables, integradas a la comunidad sin modificar notoriamente la estabilidad del sistema integrado flora-fauna. Por ello, esta concepción se asocia y converge con un concepto más amplio conocido como desarrollo sustentable, el cual es definido claramente en el capítulo "Nuestro futuro común" del Informe Brundtland (1987): El desarrollo sustentable es aquel que satisface las necesidades presentes, sin menoscabar la capacidad de las futuras generaciones de satisfacer sus propias necesidades, suponiendo un replanteo de los aspectos ecológicos, económicos, sociopolíticos y culturales. Asimismo, hace mención a un mundo humano, el cual procura respetar los recursos de la naturaleza. Desde el punto de vista ecológico, se pretende que un 
ecosistema o agroecosistema logre mantener o aumentar en el tiempo el potencial biológico y físico, que permite a su vez la reproducción de seres vivos que lo habitan y la producción de igual o mayor cantidad de bienes y servicios que los mismos generan. En cuanto a la dimensión económica, ésta se relaciona con la posibilidad de los productores de satisfacer condiciones de vida dignas, manteniendo su capital ecológico; esto conlleva un proceso lento y complejo, en el que será necesario disponer de un conjunto de incentivos, restricciones y conocimientos necesarios que deberán ser adaptados a la problemática de cada región y empresa en particular y que, además, comprende los resultados de la empresa y de los distintos componentes relacionados con ella. Por último, los aspectos sociopolíticos-culturales guardan relación con la necesidad de crear y/o fortalecer instituciones para incentivar y fortalecer la participación del sector en la promoción de una estrategia de desarrollo sustentable. Así, una empresa se considera sustentable cuando es rentable para el productor e industria asociada, aumentando la calidad de vida de las poblaciones rurales, contribuyendo con políticas de desarrollo del país y manteniendo la integridad de los recursos naturales.

Por otra parte, durante las últimas décadas, se han evidenciado una serie de tendencias que regulan las formas productivas de los países y que, paulatinamente, provocan ciertos desarreglos de índole ambiental. Estas consideraciones no escapan a la actividad forestal y, en particular, pueden resumirse en:

- El avance de la frontera agrícola y ganadera, como consecuencia de la necesidad de abrir nuevas tierras de cultivo y pastoreo para abastecimientos propios y sostener las exportaciones que dan sustento a las economías de países en desarrollo.

- La expansión demográfica de ciertos países como India, Indonesia, China y Japón se caracterizan por tener un alto nivel de vida y no disponen de tierras agrícolas (arables), con lo cual obligan a "ganar" mayores espacios, produciéndose el desmonte de tierras (en particular bosques tropicales) en lugares en donde vive la población en mayores condiciones de pobreza del mundo.

Por lo que respecta al caso de Argentina, estas fuerzas causales que actúan a favor de la deforestación mundial (pobreza, producción agrícola, producción energética e infraestructura) no tienen igual incidencia que en otros lugares del mundo. Exis- 
ten condiciones socioeconómicas, demográficas, de disponibilidad de tierra y de niveles de desarrollo que manifiestan una situación diversa. Sin embargo, la no consideración de la actividad forestal como parte de la empresa agropecuaria obedece, principalmente, a factores culturales y administrativos que - por motivos geopolíticos o socioeconómicos y por proyectos de desarrollo rural basados en la expansión de las fronteras agrícolas y ganaderas - han actuado en desmedro de esta producción.

En un sentido amplio, la silvicultura se ocupa de las acciones operadas en el bosque y sus consecuencias biológicas. Tradicionalmente, se trata de montes conformados por distintas especies, de largos periodos de aprovechamiento, cuya cosecha guarda estrecha relación con prácticas de conservación (protección del medioambiente, del suelo, de cuencas hidrográficas, de la fauna, saneamiento de la atmósfera, fuente de valores ecológicos, genéticos, paisajísticos, recreación- esparcimiento, pastaje, entre otras), más allá del rédito económico que pudiera obtenerse de su explotación. Desde este punto de vista, los bosques deben administrarse por ser recursos que, si bien son renovables, deben conservarse por sus múltiples funciones. La silvicultura se sirve de una herramienta, la ordenación forestal, la cual integra a las ciencias biológicas, sociales, económicas y todas aquellas que afectan las decisiones que hacen a la gestión física del bosque la base ecológica.

La actividad forestal está comprendida dentro de esta definición; en términos generales, forestar significa formar bosques. En ocasiones a este concepto se le relaciona con implantar bosques nuevos (cuando no existieran) o de repoblar y/ o enriquecer, sobre una extensión dada, los que estaban emplazados o aún persistieran. El uso de la forestación en sus diversas modalidades, ya sea en forma de cortinas, montes de abrigo o protectores, o, hasta en algunos casos, como pequeños macizos generarían externalidades positivas, proporcionando cambios en el micro clima, al actuar como barrera mecánica en zonas con tierras marginales carentes de capacidad de producción, además de posibilitar la incorporación de otras actividades productivas o favorecer las existentes.

\subsection{Consideraciones de las plantaciones forestales en el marco de la sustentabilidad}

Como anteriormente se señaló, en este estudio se puso especial atención al papel del productor para alcanzar, en términos sustentables, el objetivo estratégico de obtener el monte en pie y determinar los beneficios que obtendría al medir las 
externalidades positivas que se generan. En relación con el concepto de sustentabilidad puede precisarse:

- Desde la dimensión ecológica, fundamentalmente, cambios en las alternativas del uso de la tierra, favoreciendo la conservación de los suelos (capacidad productiva) y logrando un nivel de productividad sostenible;

- En la dimensión económica, una alternativa de mejora en el equilibrio del sistema productivo que resulta de incorporar la actividad silvícola a modelos agropecuarios tradicionales y de analizar las variaciones en la rentabilidad de este sistema integrado. Esto favorecería el ambiente e, indirectamente, mejoraría rendimientos de los cultivos implantados y resguardo del suelo. En casos en que se generen conflictos por el uso económico de la tierra, destinado a la agricultura, se puede establecer la forestación como sistema agroforestal;

- Por último, desde la dimensión sociopolítica-cultural, contribución a la creación de fuentes de uso de la mano de obra rural de forma más estable y con conocimientos más tecnificados, logrando un mayor y mejor asentamiento humano en cuanto a temas vinculados con la salud, la cultura y las comunicaciones.

Dentro de este marco contextual, y con el objetivo de encontrar la forma más objetiva de cuantificar las externalidades positivas y negativas de los modelos trabajados, se diseñó una matriz de evaluación y ponderación de impactos. Esta matriz, que contiene indicadores cuantitativos y cualitativos, se elaboró para que la variable medioambiente fuera incorporada en el proceso decisorio del empresario agropecuario, en términos prospectivos.

\section{Diseño de la matriz de evaluación y ponderación}

La metodología de trabajo integró conocimientos, enfoques y herramientas interdisciplinarias que permiten proponer alternativas sistémicas para la empresa rural. Se pretendió que esta propuesta contribuyera con la planificación y toma de decisiones empresariales, además de brindar información adicional para facilitar la creación de espacios alternativos de interrelación e integración entre los sectores públicos y privados, donde la sustentabilidad fuera el principio rector de esta in- 
vestigación porque la tierra es condición natural y factor indispensable de supervivencia para la actividad productiva agropecuaria.

Sabemos que el uso de indicadores es conveniente para evaluar el desempeño de las organizaciones y, así, determinar cómo está funcionando el negocio, permitiendo introducir las modificaciones que se consideren necesarias para mejorar la gestión de la actividad. Asimismo, se han tomado como referencia desarrollos teóricos actuales que tratan el impacto ambiental desde diferentes perspectivas, los cuales contemplan el empleo de indicadores.

De acuerdo con la necesidad de generar información sintética y relevante, se diseñó una matriz de evaluación y ponderación de impactos, cuyo objetivo es medir, en un único instrumento, las externalidades positivas y negativas más significativas del proyecto, incluyendo el análisis los factores cuantitativos y cualitativos que intervienen en la decisión.

\subsection{Características generales de la metodología}

El método que se utilizó, luego de analizar diversas fuentes bibliográficas sobre la medición y evaluación de impactos producidos por la puesta en marcha de un proyecto, es un híbrido, adaptado al objeto de estudio del Proyecto de Grupo de Investigación, el cual nació de dos aportes fundamentales:

1. La matriz interactiva simple o matriz de Leopold. Es una herramienta que muestra las acciones del proyecto o actividades en un eje y los factores ambientales pertinentes a lo largo del otro eje de la matriz. En la intersección de filas y columnas se apunta la magnitud y la importancia de cada uno de los efectos, que se expresan numéricamente siguiendo una escala de 1 a $5 .^{7}$

\footnotetext{
${ }^{7}$ Véase: Larry W. Canter (1999), Manual de evaluación de impacto ambiental. Técnicas para la elaboración de estudios de impacto. Universidad de Oklahoma, Capítulo 3, pp. 75-99.
} 


\begin{tabular}{|c|c|c|c|c|c|c|}
\hline & & ACCIONES / ETAPAS & $\begin{array}{l}\text { Preparación } \\
\text { del terreno }\end{array}$ & Plantación & $\begin{array}{c}\text { Mantenimiento } \\
\text { hasta planta } \\
\text { lograda }\end{array}$ & \begin{tabular}{|c|}
$\begin{array}{c}\text { Mantenimiento } \\
\text { hasta monte en } \\
\text { pie }\end{array}$ \\
\end{tabular} \\
\hline \multicolumn{7}{|c|}{ Elementos impactados } \\
\hline \multirow{21}{*}{ 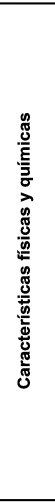 } & \multirow{4}{*}{ Tierra } & Contenido de materia orgánica del suelo & $-3 / 4$ & $0 / 0$ & $1 / 3$ & $5 / 5$ \\
\hline & & \begin{tabular}{|l|} 
Fertilidad del suelo \\
\end{tabular} & $-3 / 4$ & $0 / 0$ & $1 / 3$ & $5 / 5$ \\
\hline & & pH del suelo & $0 / 0$ & $0 / 0$ & $0 / 0$ & $-3 / 1$ \\
\hline & & Profundidad del suelo & $1 / 5$ & $0 / 0$ & $0 / 0$ & $5 / 4$ \\
\hline & \multirow{3}{*}{ Agua } & Superficial & $1 / 1$ & $0 / 0$ & $3 / 3$ & $5 / 4$ \\
\hline & & Subterránea & $1 / 1$ & $0 / 0$ & $3 / 3$ & $5 / 4$ \\
\hline & & Calidad del agua & $1 / 1$ & $0 / 0$ & $1 / 1$ & $5 / 4$ \\
\hline & \multirow{7}{*}{ Atmósfera } & Calidad del aire (partículas en suspensión) & $-1 / 1$ & $0 / 0$ & $1 / 5$ & $4 / 5$ \\
\hline & & Secuestro de dióxido de carbono & $-1 / 1$ & $1 / 5$ & $3 / 5$ & $5 / 5$ \\
\hline & & Intercambio gaseoso en la profundidad del suelo & $1 / 3$ & $1 / 5$ & $3 / 5$ & $5 / 5$ \\
\hline & & Clima (micro) & $0 / 0$ & $0 / 0$ & $1 / 1$ & $3 / 4$ \\
\hline & & \begin{tabular}{|l} 
Temperatura \\
\end{tabular} & $0 / 0$ & $1 / 1$ & $3 / 3$ & $5 / 5$ \\
\hline & & Reducción de la velocidad del viento & $-5 / 5$ & $0 / 0$ & $1 / 1$ & $5 / 5$ \\
\hline & & Humedad & $0 / 0$ & $1 / 1$ & $3 / 3$ & $5 / 5$ \\
\hline & \multirow{7}{*}{ Procesos } & Reciclado de nutrientes del suelo & $1 / 1$ & $-1 / 1$ & $1 / 1$ & $5 / 5$ \\
\hline & & \begin{tabular}{|l} 
Estructura y textura del suelo \\
\end{tabular} & $5 / 4$ & $-1 / 3$ & $3 / 4$ & $5 / 5$ \\
\hline & & Compactación & $5 / 4$ & $-1 / 1$ & $3 / 4$ & $5 / 5$ \\
\hline & & Erosión & $-1 / 1$ & $3 / 4$ & $4 / 1$ & $5 / 5$ \\
\hline & & Aireación del suelo & $1 / 2$ & $-1 / 1$ & $5 / 5$ & $5 / 5$ \\
\hline & & \begin{tabular}{|l|l|} 
Infiltración \\
\end{tabular} & $1 / 4$ & $1 / 3$ & $3 / 4$ & $5 / 4$ \\
\hline & & Reducción de pérdidas por lixiviación & $-1 / 5$ & $1 / 3$ & $3 / 4$ & $5 / 5$ \\
\hline \multirow{12}{*}{ 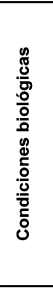 } & \multirow{3}{*}{ Flora } & Cultivos & $0 / 0$ & $1 / 1$ & $3 / 3$ & $5 / 5$ \\
\hline & & Microflora & $-1 / 1$ & $1 / 5$ & $3 / 5$ & $5 / 5$ \\
\hline & & Diversidad biológica & $-5 / 3$ & $1 / 5$ & $3 / 5$ & $5 / 5$ \\
\hline & \multirow{5}{*}{ Fauna } & Animales terrestres & $-5 / 5$ & $-5 / 3$ & $-3 / 1$ & $4 / 5$ \\
\hline & & Aves & $-1 / 1$ & $1 / 5$ & $3 / 5$ & $5 / 5$ \\
\hline & & \begin{tabular}{|l} 
Diversidad biológica \\
\end{tabular} & $-5 / 5$ & $-5 / 3$ & $-3 / 1$ & $4 / 5$ \\
\hline & & \begin{tabular}{|l} 
Microfauna (lombrices) \\
\end{tabular} & $-1 / 5$ & $3 / 5$ & $5 / 5$ & $5 / 5$ \\
\hline & & Insectos & $-5 / 4$ & $-5 / 4$ & $-5 / 4$ & $3 / 5$ \\
\hline & \multirow{4}{*}{ Usos del suelo } & Agricultura & $0 / 0$ & $0 / 0$ & $3 / 3$ & $3 / 5$ \\
\hline & & Ganadería & $0 / 0$ & $0 / 0$ & $3 / 3$ & $3 / 5$ \\
\hline & & \begin{tabular}{|l|} 
Naturaleza y espacios abiertos \\
\end{tabular} & $0 / 0$ & $0 / 0$ & $1 / 3$ & $1 / 3$ \\
\hline & & Comercial & $0 / 0$ & $0 / 0$ & $0 / 0$ & $1 / 3$ \\
\hline \multirow{3}{*}{ 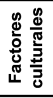 } & Estética e interés humano & Vistas escénicas y panorámicas & $-1 / 1$ & $-2 / 1$ & $3 / 3$ & $3 / 5$ \\
\hline & \multirow{2}{*}{ Estatus cultural } & Empleo & $1 / 1$ & $1 / 1$ & $0 / 0$ & $0 / 0$ \\
\hline & & Pautas culturales (estilo de vida rural) & $1 / 1$ & $1 / 1$ & $3 / 5$ & $4 / 5$ \\
\hline \multirow{3}{*}{ 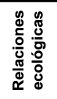 } & & Salinización de recursos hídricos & $0 / 0$ & $0 / 0$ & $0 / 0$ & $5 / 5$ \\
\hline & & Eutrofización & $0 / 0$ & $0 / 0$ & $0 / 0$ & $1 / 5$ \\
\hline & & Invasiones de maleza & $0 / 0$ & $0 / 0$ & $0 / 0$ & $-2 / 5$ \\
\hline
\end{tabular}

2. La propuesta del Gobierno de la Nación a través de la Ley Nacional $N^{o}$ $25080 / 98$ y el Decreto Reglamentario 133/99. En ellos se plantea una matriz de impactos, en la que de acuerdo con una serie de actividades (dispuestas en columnas) y de recursos (dispuestos en filas) valora los impactos acorde a una tipificación establecida. ${ }^{8}$

Los criterios para elegir esta herramienta se basaron principalmente en:

- Existencia de datos cuantitativos en la utilización de este instrumento, eliminando los métodos que sólo hacen comparaciones descriptivas o cualitativas.

${ }^{8}$ Véase: Ley Nacional N 25080/98 y Decreto Reglamentario 133/99. 
- Claridad en la exposición de la información.

- Posibilidad de realizar comparaciones entre los distintos resultados obtenidos (por ejemplo: impactos positivos versus impactos negativos).

- Oportunidad de "objetivizar" la medición de los impactos a través de un debate interdisciplinario.

Por otra parte, se efectuó una adaptación de la matriz original de Leopold, como aporte metodológico para alcanzar el objetivo general del proyecto, ante la necesidad de:

- Contemplar un mayor espectro de indicadores económicos y sociopolítico -cultural en la evaluación de impactos.

- Considerar la promoción de una herramienta similar por parte del sector público de nuestro país, cuya aplicación es requerida para solicitar los subsidios estipulados en la Ley Nacional $N^{\circ}$ 25080/98.

\subsection{Etapas para el diseño de la matriz}

1) Se identificaron diferentes aspectos claves del proyecto por evaluar representativos de aquellos impactos potenciales críticos que la forestación generará en el desempeño sustentable de la empresa rural en sus tres dimensiones, tomando en cuenta las influencias recíprocas entre ésta y su ambiente. Se entiende por impacto a la consecuencia de una acción o actividad que produce una alteración, favorable o desfavorable, en el medio o en alguno de los componentes del medio, tanto físico como socioeconómico. ${ }^{9}$

2) Se agruparon desde las perspectivas externa e interna para contemplar dicha interacción dinámica (inputs y outputs del sistema abierto "empresa" propios del desarrollo de un nuevo proceso: el manejo forestal integrado a un planteo agrícola-ganadero). Se concibieron las alteraciones que en la situación inicial se producirían a consecuencia de la realización del proyecto; asimismo, se incluyeron los efectos directos e indirectos.

\footnotetext{
${ }^{9}$ Véase: Vicente Conesa Fernández-Vítora (1997), Guía metodológica para la evaluación del impacto ambiental, Capítulo 1, pp. 21-25.
} 
3) Se eligieron indicadores representativos de cada área clave impactada o impactante, y se procedió a su descripción, indicando para cada uno el área de evaluación (ambiental, económica y sociopolítica-cultural). ${ }^{10}$

4) Se calcularon los indicadores cuantitativos y se califican los cualitativos. ${ }^{11}$

5) Se ponderaron los resultados a partir del armado de una tabla de ponderaciones con el propósito de llevarlos a una unidad homogénea que permita la integración de todos los indicadores descritos.

\subsection{Ponderación de resultados}

\subsubsection{Definición de atributos}

Para la ponderación se valoraron los resultados en función de los siguientes atributos ${ }^{12}$ :

- Plazo: periodo durante el cual se manifiesta el impacto (corto, mediano o largo)

- Persistencia: repercusión en el tiempo de los efectos que produce el impacto y consecuencias residuales o perdurables en el medio (transitorio o permanente)

- Magnitud: modificación baja, media o alta en las condiciones del medio.

- Dirección: negativa o positiva, en caso que el efecto disminuya o aumente respectivamente el bienestar o calidad de vida de las generaciones futuras, $\mathrm{o}$ cuando va en detrimento (o mejore) el agronegocio del productor.

\subsubsection{Valoración de atributos. Tabla de ponderaciones}

La valoración de los atributos se logró a partir de la aplicación de una tabla de ponderaciones. Para la confección e implementación de nuestra matriz se tomó como referencia la opinión interdisciplinaria de expertos mediante el método Delphi y de la técnica de grupo nominal.

10 Véase: R. Durán y L. Scoponi (2003), Matriz de desempeño sustentable: una propuesta metodológica e instrumental para evaluar la sustentabilidad de la empresa agropecuaria, X Jornadas Nacionales de la Empresa Agropecuaria, pp. 23-44.

${ }^{11}$ Véase: R. Durán et al. (2003). Tablero de Comando: Una herramienta para el control de gestión de empresas agropecuarias, p. 31.

12 Véase Vicente Conesa Fernández-Vítora (1997), op. cit., pp. 29-43 y 88-97. 
En la elaboración de la tabla de ponderaciones se consideró una puntuación en la escala de 1 a 5 para asignar los valores que puede asumir cada atributo. Una vez obtenidos, se ponderaron (multiplicaron) a partir de la importancia relativa otorgada a cada uno de ellos. El signo positivo o negativo representará la dirección del efecto.

\subsection{Formato de la matriz}

Debido a lo anterior, el diseño de la matriz tuvo el siguiente formato:

- Las filas representan los impactos detectados.

- Las columnas describen el tipo de impacto de que se trate.

- Las celdas son la intersección que describe qué características posee cada tipo de impacto.

En cuanto a los indicadores, éstos se agruparon desde dos perspectivas: interna y externa. A continuación se detalla cada una.

\subsubsection{Perspectiva interna}

Se identificaron en primer lugar aquellos indicadores relacionados directamente con el impacto de la producción forestal en el ambiente; en segundo término, los que se originan a partir del análisis de la actividad principal, la complementaria y el gerenciamiento.

- Indicadores del impacto de la actividad forestal en el ambiente: Se evaluaron indicadores atmosféricos, edáficos, hídricos, de procesos relativos a las características físicas y químicas del suelo, el aire y el agua; además de ecológicos y paisajísticos, los cuales afectan en el mediano y largo plazo la calidad de vida de nuestro planeta.

- Indicadores de la actividad principal:Se analizó cómo impactó en las actividades agropecuarias tradicionales de la empresa (agricultura, ganadería o mixta) el desarrollo de la actividad forestal como complementaria de la misma.

- Indicadores de la actividad complementaria: En este caso, los indicadores analizados se refieren a los efectos directos del desarrollo de la actividad forestal, entendido como el incremento en ingresos, consideraciones en 
costos y erogaciones relacionadas con inversiones requeridas para llevar a cabo dicha actividad. También se incluyó el uso de la infraestructura o capacidad instalada (superficie disponible).

- Indicadores de gerenciamiento: Se refiere a aquellos aspectos relacionados con la toma de decisiones en la gestión de la empresa agropecuaria en relación con la incorporación de la forestación como actividad complementaria.

\subsubsection{Perspectiva externa}

Los indicadores se vincularon con aspectos del entorno que pueden afectar a la empresa y/o ser afectados por decisiones que ésta adopte. Aparecen indicadores cualitativos para los cuales el impacto no se midió en valores sino en grados que serán especificados de acuerdo con el aspecto por medir.

Para el análisis del contexto, se estableció una delimitación de distintos subcontextos: el sociocultural, el político- legal- económico y el tecnológico; aunque principalmente de la tecnología e innovación, los cambios en modas o hábitos culturales de consumidores y la acción y reacción de los distintos actores involucrados: la población o comunidad asentada en cercanías del establecimiento, el gobierno (nacional, provincial, municipal) y los agentes ubicados en los distintos eslabones de las cadenas agroforestales.

\subsubsection{Matriz de impactos}

A continuación se presenta el formato de la matriz en la cual se exhiben los indicadores ponderados y sus perspectivas analizadas. El intervalo de soluciones factibles para la matriz es $=[-355 ; 355]$. En el intervalo $[-355 ; 0)$, los impactos negativos exceden a los positivos. En el intervalo $(0 ; 355]$ sucede lo contrario, es decir, los impactos positivos superan a los negativos. Si el resultado de la matriz es = 0 implica que la valoración de los impactos positivos es igual a la de los impactos negativos. 


\begin{tabular}{|c|c|c|c|c|c|c|}
\hline INDICADORES & No. & Plazo & $\mathbf{P}$ & M & D & $\begin{array}{c}\text { Ponderación } \\
\text { final }\end{array}$ \\
\hline PERSPECTIVA INTERNA & & h+1+H+h+h & h+1+h+1+t+h+t & h+1+h+h+1+h+t & h+h+h+h+1+ht+h & h+1+h+1+h+h+t \\
\hline $\begin{array}{l}\text { Indicadores del impacto de la actividad forestal } \\
\text { en el ambiente }\end{array}$ & & h+1+11+1+ & h+111+1+1h+h+t & $h+1+1+1+1+1+1+$ & $h+1+1+1+1+1+1+$ & 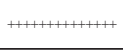 \\
\hline Respecto a la atmósfera & & H+h+h+ht & h+h+1+1+h+t+t & 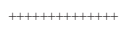 & 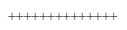 & h+h+h+h+h+th \\
\hline Secuestro de dióxido de carbono atmosférico (CO2) & 1 & 5 & 5 & 5 & 1 & 5 \\
\hline Fijación de nitrógeno atmosférico (N2) & 2 & 5 & 1 & 5 & 1 & 3,2 \\
\hline Reducción de la velocidad del viento & 3 & 1 & 5 & 5 & 1 & 3,6 \\
\hline Calidad del aire (partículas en suspensión) & 4 & 1 & 5 & 3 & 1 & 3,2 \\
\hline Intercambio gaseoso en la profundidad del suelo & 5 & 3 & 5 & 3 & 1 & 3,9 \\
\hline Clima (micro) & 6 & 1 & 5 & 1 & 1 & 2,8 \\
\hline Temperatura & 7 & 1 & 5 & 3 & 1 & 3,2 \\
\hline Humedad & 8 & 1 & 5 & 3 & 1 & 3,2 \\
\hline Respecto al componente edáfico & & H+h+1+H+h & H+11+1+1+1+1+ & 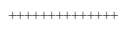 & 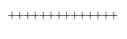 & H+1+1+1+1+1+1+th \\
\hline Agua superficial & 9 & 1 & 5 & 5 & 1 & 3,6 \\
\hline Agua subterránea & 10 & 1 & 5 & 5 & 1 & 3,6 \\
\hline Calidad del agua & 11 & 1 & 5 & 5 & 1 & 3,6 \\
\hline Contenido de materia orgánica & 12 & 1 & 5 & 5 & 1 & 3,6 \\
\hline Fertilidad del suelo & 13 & 1 & 5 & 3 & 1 & 3,2 \\
\hline Humedad del suelo & 14 & 1 & 5 & 5 & 1 & 3,6 \\
\hline PH del suelo & 15 & 1 & 1 & 3 & -1 & $-1,4$ \\
\hline Profundidad del suelo & 16 & 1 & 5 & 3 & 1 & 3,2 \\
\hline Temperatura del suelo & 17 & 1 & 5 & 5 & 1 & 3,6 \\
\hline Compactación del suelo & 18 & 1 & 5 & 5 & 1 & 3,6 \\
\hline Estructura y textura del suelo & 19 & 1 & 5 & 5 & 1 & 3,6 \\
\hline Respecto a los procesos & & $h+1+1+1+1+$ & $h+1+1+1+1+1+1+$ & $h+H+H+H+h+H$ & $h+1+1+1+h+1+1+$ & $h+H+1+H+h+H+t$ \\
\hline Reciclado de nutrientes del suelo & 20 & 1 & 5 & 3 & 1 & 3,2 \\
\hline Erosión & 21 & 1 & 5 & 3 & 1 & 3,2 \\
\hline Aireación del suelo & 22 & 1 & 5 & 5 & 1 & 3,6 \\
\hline Infiltración del agua & 23 & 1 & 5 & 5 & 1 & 3,6 \\
\hline Reducción de pérdidas por lixiviación & 24 & 1 & 5 & 3 & 1 & 3,2 \\
\hline Respecto a las condiciones biológicas & & H+H+1H+r & H+H+1+1+11+1+ & H+H+H+H+H & $h+1+1+1+1+1+1+$ & H+H+t+1+H+t+ \\
\hline Flora & & H+1+1+1+ & H+11+1+1+1+1+t+ & $H+1+1+1+1+1+$ & $h+1+1+1+1+1+1+$ & H+1+1+1+1+1+1+ \\
\hline Cultivos & 25 & 1 & 5 & 3 & 1 & 3,2 \\
\hline Microflora & 26 & 5 & 5 & 3 & 1 & 4,6 \\
\hline Diversidad biológica & 27 & 5 & 5 & 3 & 1 & 4,6 \\
\hline Fauna & & H+t+1+1+r & H++1+1+1+1+1+t & $H+1+H+1+1+H$ & $h+1++1+1+1+1+$ & H+1+1+1+1+1+t+ \\
\hline Animales terrestres & 28 & 5 & 1 & 3 & -1 & $-2,8$ \\
\hline Aves & 29 & 3 & 5 & 3 & 1 & 3,9 \\
\hline Diversidad biológica & 30 & 5 & 1 & 3 & -1 & $-2,8$ \\
\hline Microfauna (lombrices) & 31 & 1 & 5 & 3 & 1 & 3,2 \\
\hline Insectos & 32 & 3 & 5 & 3 & -1 & $-3,90$ \\
\hline Usos del suelo & & H+h+1hH+ & H+11+1+1+1+1+t+ & $H+1+1+1+1+1+$ & $h+1+1+1+1+1+1+$ & H+1+1+1+1+1+1+ \\
\hline Agricultura & 33 & 3 & 5 & 3 & 1 & 3,9 \\
\hline Ganadería & 34 & 3 & 5 & 3 & 1 & 3,9 \\
\hline Naturaleza y espacios abiertos & 35 & 3 & 5 & 1 & 1 & 3,5 \\
\hline Comercial & 36 & 1 & 5 & 1 & 1 & 2,8 \\
\hline Respecto a las relaciones ecológicas & & hH+h+h+ht+h & 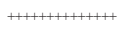 & 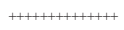 & $h+h+1+h+h+1+t$ & 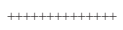 \\
\hline Salinización de recursos hídricos & 37 & 1 & 5 & 5 & 1 & 3,6 \\
\hline Eutrofización & 38 & 1 & 5 & 1 & 1 & 2,8 \\
\hline Invasiones de maleza & 39 & 1 & 5 & 3 & -1 & $-3,2$ \\
\hline
\end{tabular}




\begin{tabular}{|c|c|c|c|c|c|c|}
\hline INDICADORES & $\mathbf{N}^{0}$ & Plazo & $\mathbf{P}$ & $\mathbf{M}$ & $\mathbf{D}$ & Ponderación final \\
\hline Indicadores de la actividad principal & & +++++++++ & ++++++++++++ & +++++++++++++ & +++++++++++++ & +++++++++++++ \\
\hline Respecto a la producción & & ++++++++++ & $t+++++++++1+++$ & ++++++++++++ & ++++++++++++++ & ++++++++++++++ \\
\hline Productividad agrícola & 40 & 1 & 5 & 3 & 1 & 3,2 \\
\hline Productividad ganadera & 41 & 1 & 5 & 3 & 1 & 3,2 \\
\hline Respecto a los costos & & ++++++++++ & $+++++1++++1+++$ & ++++++++++++++ & ++++++++++++ & ++++++++++++++ \\
\hline Ahorro en costos de alimentación & 42 & 1 & 5 & 3 & 1 & 3,2 \\
\hline Ahorro en costos de fertilización & 43 & 1 & 5 & 3 & 1 & 3,2 \\
\hline Indicadores de la actividad complementaria & & ++++++++++ & +++++++++++++ & ++++++++++++++ & ++++++++++++++ & ++++++++++++++ \\
\hline Respecto a los ingresos & & ++++++++++ & +++++++++++++++ & ++++++++++++++ & ++++++++++++++ & ++++++++++++++ \\
\hline Venta de leña y postes & 44 & 1 & 5 & 1 & 1 & 2,8 \\
\hline $\begin{array}{l}\text { Respecto a la inversión necesaria hasta } \\
\text { alcanzar la planta lograda }\end{array}$ & & +++++++++ & ++++++++++++++ & ++++++++++++++ & ++++++++++++++ & ++++++++++++++ \\
\hline Inversión de capital circulante con subsidio & 45 & 3 & 1 & 3 & -1 & $-2,10$ \\
\hline Inversión de capital circulante sin subsidio & 46 & 3 & 1 & 5 & -1 & $-2,50$ \\
\hline $\begin{array}{l}\text { Valuación del campo } \\
\end{array}$ & 47 & 3 & 5 & 5 & 1 & 4,30 \\
\hline $\begin{array}{r}\text { Costos fijos operativos de mantenimiento hasta } \\
\text { alcanzar la planta lograda }\end{array}$ & 48 & 3 & 1 & 3 & -1 & $-2,10$ \\
\hline Costo de capital invertido & 49 & 3 & 1 & 1 & -1 & $-1,70$ \\
\hline $\begin{array}{r}\text { Costo de mano de obra especializada en } \\
\text { actividades forestales }\end{array}$ & 50 & 5 & 1 & 3 & -1 & $-2,80$ \\
\hline $\begin{array}{r}\begin{array}{r}\text { Costo de asesoramiento profesional en la } \\
\text { actividad }\end{array} \\
\end{array}$ & 51 & 5 & 1 & 1 & -1 & $-2,40$ \\
\hline Costo de capacitación & 52 & 5 & 1 & 1 & 1 & 2,40 \\
\hline $\begin{array}{l}\text { Respecto a los costos de mantenimiento desde } \\
\text { la planta lograda } \\
\text { hasta obtener el monte de pie }\end{array}$ & & +++++++++ & ++++++++++++++ & ++++++++++++++ & ++++++++++++++ & ++++++++++++++ \\
\hline Costos de capital invertido & 53 & 3 & 1 & 1 & -1 & $-1,70$ \\
\hline $\begin{array}{r}\text { Costos fijos operativos de mantenimiento de } \\
\text { la forestación }\end{array}$ & 54 & 3 & 5 & 3 & 1 & 3,90 \\
\hline $\begin{array}{r}\text { Costo de mano de obra especializada en } \\
\text { actividades forestales }\end{array}$ & 55 & 3 & 5 & 3 & 1 & 3,90 \\
\hline $\begin{array}{r}\text { Costo de asesoramiento profesional en la } \\
\text { actividad }\end{array}$ & 56 & 3 & 5 & 1 & 1 & 3,50 \\
\hline $\begin{array}{l}\text { Respecto al aprovechamiento de la } \\
\text { infraestructura }\end{array}$ & & +++++++++ & ++++++++++++ & +++++++++++++ & +++++++++++++ & ++++++++++++ \\
\hline Aprovechamiento de la superficie & 57 & 1 & 5 & 1 & 1 & 2,80 \\
\hline Planificación en el uso de terreno & 58 & 1 & 5 & 3 & 1 & 3,20 \\
\hline Indicadores de gerenciamiento & & ++++++++++ & ++++++++++++++ & ++++++++++++++ & ++++++++++++++ & ++++++++++++++ \\
\hline Definición de la misión y estrategia & 59 & 5 & 5 & 5 & -1 & $-5,00$ \\
\hline Organización & 60 & 3 & 5 & 5 & -1 & $-4,30$ \\
\hline PERSPECTIVA EXTERNA & & +++++++++ & +++++++++++++ & ++++++++++++++ & ++++++++++++++ & ++++++++++++++ \\
\hline Subcontexto sociocultural & & ++++++++++ & $++++++1+++1+++$ & ++++++++++++++ & +++++++++++++ & ++++++++++++++ \\
\hline Pautas culturales (estilo de vida rural) & 61 & 5 & 5 & 5 & -1 & $-5,00$ \\
\hline Empleo & 62 & 5 & 1 & 1 & 1 & 2,40 \\
\hline Visitas escénicas y panorámicas & 63 & 1 & 5 & 3 & 1 & 3,20 \\
\hline Protección del medioambiente & 64 & 5 & 1 & 5 & -1 & $-3,20$ \\
\hline Subcontexto político-legal y económico & & +++++++++ & ++++++++++++++ & ++++++++++++++ & ++++++++++++++ & +++++++++++++ \\
\hline Gestión de politica ambiental forestal & & +++++++++ & ++++++++++++++ & ++++++++++++++ & ++++++++++++++ & ++++++++++++++ \\
\hline Legislación disponible & 65 & 5 & 5 & 3 & 1 & 4,60 \\
\hline Accesibilidad al crédito & 66 & 5 & 1 & 3 & -1 & $-2,80$ \\
\hline Incentivos forestales & 67 & 5 & 5 & 5 & 1 & 5,00 \\
\hline Subsidio & 68 & 3 & 1 & 5 & 1 & 2,50 \\
\hline Existencia de viveros & 69 & 5 & 5 & 3 & 1 & 4,60 \\
\hline Subcontexto tecnológico & & ++++++++++ & ++++++++++++++ & +++++++++++++ & +++++++++++++ & ++++++++++++++ \\
\hline $\begin{array}{r}\text { Avances en el desarrollo de cultivos para zonas } \\
\text { marginales } \\
\text { (competencia con forestación) }\end{array}$ & 70 & 1 & 1 & 5 & 1 & 1,80 \\
\hline $\begin{array}{r}\text { Avances en el desarrollo de especies forestales, } \\
\text { aptas para zonas marginales }\end{array}$ & 71 & 1 & 1 & 1 & -1 & $-1,00$ \\
\hline TOTAL & & & & & & 133,10 \\
\hline
\end{tabular}




\section{Discusión de resultados}

Del diagnóstico estratégico efectuado para determinar cuál era el estado de situación actual de las empresas agropecuarias en términos de sustentabilidad, se observaron efectos de la degradación del recurso suelo producto de una visión cortoplacista en el gerenciamiento del sector. A partir de la identificación de aquellos suelos, con serias limitantes para el desarrollo de las actividades agropecuarias tradicionales (pedregosidad, salinidad, etc.), se proponen modelos de implantación de especies que, como actividad complementaria, contribuyan a la sostenibilidad del sistema evitando un círculo vicioso de degradación.

Después de la evaluación y ponderación de impactos mediante la aplicación de una matriz de indicadores de sustentabilidad ecológica, económica y sociopolítica-cultural diseñada ad hoc, se corrobora la hipótesis planteada, debido a que los resultados del impacto de las externalidades positivas superan ampliamente los efectos negativos (ponderación final equivalente a 133,10), por lo que se propone la forestación como una alternativa para mejorar la sostenibilidad de los sistemas productivos tradicionales de la Pampa semiárida argentina en tierras marginales, sin olvidar que el propósito no está enfocado en su abordaje como actividad comercial, sino como una alternativa adicional y sustentable.

De igual forma, se observa que los impactos que la decisión provoca en las necesidades de inversión hasta alcanzar la planta lograda son bajos, siendo altamente positiva la incidencia de la revalorización final que causa el manejo propuesto en las tierras marginales degradadas de la región bajo estudio, aspecto no contemplado en la matriz original de Leopold. Asimismo, los costos de mantenimiento desde la planta lograda hasta obtener el monte en pie tienen en su mayoría una incidencia positiva significativa, lo que se debe a que por ser poco importantes no representan un obstáculo para el productor.

Por otra parte, no se han encontrado avances tecnológicos que puedan plantear alternativas de cultivos a la forestación con alta factibilidad para mejorar la sustentabilidad de los agroecosistemas de tierras marginales en el Partido de Bahía Blanca.

En cuanto al balance de los impactos de la actividad forestal en el ambiente, éste es altamente positivo, dado que la superficie que se propone afectar con la intervención no supera el $20 \%$ del total. Además, se trata de tierras que en ningún caso se destinarán a reservas naturales por ser ambientes ya degradados. 
Cabe destacar que las evaluaciones negativas se han correspondido con aspectos que hacen a la cultura de las empresas agropecuarias de la región bajo estudio y a la idiosincrasia del sector. Esto empeora por la carencia de políticas públicas activas que favorezcan el desarrollo sustentable de la actividad rural, particularmente en lo que se refiere a su continuidad en el tiempo, simplicidad para el productor y pragmatismo en su implementación que eviten su limitación al ámbito meramente discursivo. El efecto de la gestión de política ambiental forestal es en principio positivo, dada la existencia de legislación, incentivos forestales y viveros públicos que crean condiciones favorables.

\section{Conclusiones}

La administración de la forestación se plantea como una posibilidad para mitigar los impactos de la producción primaria en tierras marginales relacionada con la compactación, la erosión y la disminución de la humedad del suelo. Estas externalidades en la dimensión ambiental potenciarán en el mediano plazo los otros ejes en los que se funda la sustentabilidad. En cuanto al aspecto económico, aumentan los rendimientos físicos de las actividades principales (producción de cultivos y de ganado bovino) y se ahorran ciertos costos de producción, entre otros efectos. Por lo que respecta al pilar sociopolítico-cultural, contribuiría a evitar el éxodo rural, pues se encontrarían nuevas fuentes de desarrollo del capital humano, base del capital social.

Si bien se concibe que la empresa agropecuaria debe ser socialmente responsable en su desempeño, al seleccionar aquellos cursos de acción que promuevan la sustentabilidad del agroecosistema, el rol del Estado no debe estar ausente en esta problemática, por tratarse de una responsabilidad compartida inherente al bien común.

La adopción de políticas públicas activas y estables de largo plazo, con una visión estratégica orientada al desarrollo agropecuario sustentable, disiparía la incertidumbre que percibe el empresario rural para incorporar la silvicultura en su sistema de producción como alternativa complementaria. Favorecerían paulatinamente el cambio cultural y la rápida adopción de decisiones como las que se proponen, que técnica y económicamente son factibles, pudiendo así estar en concordancia con las exigencias que impone el mundo moderno no sólo de competitividad, sino también las relativas a la conservación del capital natural y desarrollo del capital social. 


\section{Bibliografía}

CANTER, Larry W. (1999), Manual de evaluación de impacto ambiental. Técnicas para la elaboración de estudios de impacto, $2^{\mathrm{a}}$, ed., Universidad de Oklahoma, McGraw Hill.

Conesa Fernández-Vítora, Vicente (1997), Guía metodológica para la evaluación del impacto ambiental, $3^{\mathrm{a}}$, ed., ediciones Mundi-Prensa.

DURÁn R. et al., (2003). Tablero de Comando: Una herramienta para el control de gestión de empresas agropecuaria, Osmar Buyatti Librería Editorial, Buenos Aires.

y L. SCOPOnI (2003), Matriz de desempeño sustentable: una propuesta metodológica e instrumental para evaluar la sustentabilidad de la empresa agropecuaria, X Jornadas Nacionales de la Empresa Agropecuaria, Osmar Buyatti, Buenos Aires.

y et al. (2005). El gerenciamiento agropecuario en el siglo XXI. Bases para una competitividad sustentable, Osmar Buyatti, Buenos Aires.

Gallo, Miguel A. (1992), "Responsabilidades sociales dentro y fuera de la empresa, Estrategia y sociedad: responsabilidades sociales", Enciclopedia de Dirección y Administración, ORBIS, 67.

Kent, Patricia (2005), "Cambios en la teoría y tecnologías administrativas", en Miguel A. Vicente et al., Fundamentos de administración de organizaciones, La Ley, Buenos Aires, pp. 374 (Colección Académica). 